\title{
AXIAL MAPS WITH FURTHER STRUCTURE
}

\author{
A. J. BERRICK
}

\begin{abstract}
For $F=\mathbf{R}, \mathbf{C}$ or $\mathbf{H}$ an $F$-axial map is defined to be an axial map $\mathbf{R} P^{m} \times \mathbf{R} P^{m} \rightarrow \mathbf{R} P^{m+k}$ equivariant with respect to diagonal and trivial $F^{*}$-actions. Analogously to the real case, it is shown that $\mathbf{C}$-axial maps correspond to immersions of $\mathbf{C} P^{n}$ in $\mathbf{R}^{2 n+k}$ while (for $F=\mathbf{R}$ and for $F=\mathbf{C}$, $k$ odd) embeddings induce $F$-symmaxial maps. Examples are thereby given of symmaxial maps not induced by embeddings of $\mathbf{R} P^{n}$, and of $\mathbf{R}$-axial maps which are not $\mathbf{C}$-axial. Furthermore, the relationships which hold when $F=\mathbf{R}, \mathbf{C}$ are no longer valid for $F=\mathbf{H}$.
\end{abstract}

Let $F$ be one of the fields $\mathbf{R}, \mathbf{C}$ or $\mathbf{H}$ of dimension $d$ (=1,2,4 respectively) over $\mathbf{R}$, whose units $F^{*}$ act on the right on $S\left(F^{n+1}\right)$ to induce the projective space $F P^{n}$. Since the action of $\mathbf{R}^{*}$ extends to the action of $F^{*}$, we may regard $F^{*}$ as acting also on $\mathbf{R} P^{n}$ and thence diagonally on $\mathbf{R} P^{n} \times \mathbf{R} P^{n}$, $n \equiv-1(d)$. By way of generalisation of the usual definitions ( $F=\mathbf{R}$-see [2], [4], [12]), we say $f: \mathbf{R} P^{n} \times \mathbf{R} P^{n} \rightarrow \mathbf{R} P^{n+k}$ is $F$-axial of type $(n, k)$ if $f$ restricts to homotopy essential maps on the axes of the product and is equivariant with respect to the above $F^{*}$-action on its domain and trivial $F^{*}$-action on its range. If further $f$ is homotopy equivariant-through an $F^{*}$-equivariant homotopy-with respect to interchanging the factors of the domain and trivial $\mathbf{Z}_{2}$-action on the range, $f$ is $F$-symmaxial. (When $F=\mathbf{R}$ it is sometimes omitted from the notation.) This note explores the relationship between $F$-axial (resp. $F$-symmaxial) maps and the existence of an immersion (resp. embedding) of $F P^{n}$ in $\mathbf{R}^{m}$, denoted $F P^{n} \subseteq(m)\left(\right.$ resp. $\left.F P^{n} \subset(m)\right)$.

1. THEOREM. Let $F=\mathbf{R}$ or $\mathbf{C}$, with $N=n$ or $(2 n+1)$ respectively.

(a) If $F P^{n} \subseteq(d n+k)$, then there exists an $F$-axial map of type $(N, k)$.

(b) If $F P^{n} \subset(d n+k)$, then there exists an $F$-symmaxial map of type $(N, k)$, provided $k$ is odd if $F=\mathbf{C}$.

(c) If $F P^{n} \subset(d n+k)$, then the $F$-axial maps given by the constructions of (a) and (b) are homotopic through an $F^{*}$-equivariant homotopy.

(d) If there exists an F-axial map of type $(N, k)$ with $2 k \geqslant d n+1$, then $F P^{n} \subseteq(d n+k)$.

Proof. (a),(d). Let $\gamma$ be the realisation of the Hopf line bundle, $\varepsilon$ the trivial real line bundle, and $\tau$ the real tangent bundle over $F P^{n}$. In the following sequence of implications, ${ }^{\dagger}$ indicates the use of the condition $2 k \geqslant d n+1$.

Received by the editors December 30, 1974.

AMS (MOS) subject classifications (1970). Primary 57D40; Secondary 55D99, 55F25, 55F50.

Key words and phrases. Axial map, embedding, immersion, projective space, skew map, symmaxial map, tangent bundle. 


$$
\begin{aligned}
F P^{n} \subseteq(d n+k) \Leftrightarrow & \tau \text { is a subbundle of }(d n+k) \varepsilon[6] \\
\Leftrightarrow & \tau \oplus d \varepsilon=(n+1) \gamma^{*} \text { is a subbundle of } \\
& (d n+k+d) \varepsilon[7, \text { p. 100] } \\
\dagger \Leftrightarrow \text { there exists a skew map } & (n+1) \gamma^{*} \rightarrow(d(n+1)+k) \varepsilon[5,(1.2)] \\
\Leftrightarrow & \text { there exists a map } S^{N} \times S^{N} \rightarrow S^{N+k} \text { which induces } \\
& \text { an } F \text {-axial map of type }(N, k) .
\end{aligned}
$$

(b) Let $f: F^{P} \rightarrow \mathbf{R}^{d n+k}$ be an embedding. (To use conventional matrix notation, we shall assume here that $F^{*}$ acts on $\mathbf{R}^{d n}$ on the left.) Write $\mathbf{R}_{0}^{m}=\mathbf{R}^{m} \backslash\{0\} ; \quad \nu: \mathbf{R}_{0}^{m} \rightarrow S^{m-1}, \quad x \mapsto x /\|x\| ; \pi: S^{N} \rightarrow F P^{N}$, and set $\bar{\Delta}=\left\{(x, w x) \in \mathbf{R}_{0}^{N+1}: w \in F^{*}\right\}, \Delta^{\prime}=\bar{\Delta} \cap\left(S^{N} \times S^{N}\right), e=(1,0, \ldots, 0) \in$ $\mathbf{R}^{N+1+k}$, and $j: \mathbf{R}^{d n+k} \rightarrow \mathbf{R}^{d} \oplus \mathbf{R}^{d n+k}$ for the inclusion of the orthogonal complement of $F e$ in $\mathbf{R}^{N+1+k}$. For $u, v \in S^{N}$, write $a=\langle v, u\rangle_{F}$; and define

$$
\begin{gathered}
G:\left(S^{N} \times S^{N}, S^{N} \times S^{N} \backslash \Delta^{\prime}\right) \times I \rightarrow\left(\mathbf{R}^{N+1} \times \mathbf{R}^{N+1}, \mathbf{R}_{0}^{N+1} \times \mathbf{R}_{0}^{N+1} \backslash \bar{\Delta}\right), \\
G(u, v, t)=\left[\begin{array}{cc}
1-|a|^{2} t^{2} & -\bar{a} t \\
a t & 1
\end{array}\right]\left[\begin{array}{c}
u \\
v-a u
\end{array}\right] ; \\
g:\left(\mathbf{R}^{N+1} \times \mathbf{R}^{N+1}, \mathbf{R}_{0}^{N+1} \times \mathbf{R}^{N+1} \backslash \bar{\Delta}\right) \rightarrow\left(\mathbf{R}^{d n+k}, \mathbf{R}_{0}^{d n+k}\right), \\
g(x, y)=\left\{\begin{array}{rr}
\|x\| \cdot\|y\| \cdot\|f \pi \nu(x)-f \pi \nu(y)\| \cdot[f \pi \nu(x+y)-f \pi \nu(x-y)], \\
0, & (x, y) \in \mathbf{R}_{0}^{N+1} \times \mathbf{R}_{0}^{N+1} \backslash \bar{\Delta}
\end{array}\right.
\end{gathered}
$$

Hence, define

$$
F: S^{N} \times S^{N} \times I \rightarrow S^{N+k}, \quad F(u, v, t)=\nu(a e+j g G(u, v, t)) .
$$

The reader may verify that these maps behave as required, so that $F_{0}: S^{N} \times$ $S^{N} \rightarrow S^{N+k}$ induces an $F$-symmaxial map of type $(N, k)$. (When $F=\mathbf{C}$, the involution on $\mathbf{R} P^{2 n+1+k}$ given by $\pm(a e+j(z)) \mapsto \pm(\bar{a} e+j(z))$ is homotopic to the identity provided $k$ is odd.)

(c) Clearly it suffices to establish that the tangent bundle monomorphism

$$
\tau(f): \tau F P^{n} \rightarrow \tau \mathbf{R}^{d n+k}=\mathbf{R}^{d n+k} \times \mathbf{R}^{d n+k}
$$

is fibre-homotopic to

$$
g^{\prime}: \tau F P^{n} \rightarrow \mathbf{R}^{d n+k} \times \mathbf{R}^{d n+k}, \quad g^{\prime}\left([x, y] . F^{*}\right)=(f \pi(x), g(x, y))
$$

( $f, g$ as in (b)), since the $F$-axial maps of both (a) and (b) come from composition with $G_{0}: S^{N} \times S^{N} \rightarrow \pi^{*} \tau F P^{n}$ specified in (b).

But this is evident from the following homotopy (cf. [5, Lemma 2.2]):

$$
H: \tau F P^{n} \times I \rightarrow \mathbf{R}^{d n+k} \times \mathbf{R}^{d n+k},
$$

$$
\begin{aligned}
& H\left([x, y] \cdot F^{*}, t\right) \\
& \quad=\left(f \pi(x),[f \pi \nu(x+(1-t) y)-f \pi \nu(x-(1-t) y)] /\left(1-t^{2}\right)\right) .
\end{aligned}
$$

(Note that, as $t \rightarrow 1,1-t^{2}=2(1-t)+O\left((1-t)^{2}\right)$.)

By [2], the numerical condition of $1(\mathrm{~d})$ is satisfied when $n>7$ if $F=\mathbf{C}$ and may be omitted if $F=\mathbf{R}$. Thus $1(\mathrm{a})$,(d) yield that $\mathbf{C} P^{n} \subseteq(2 n+k)$ implies 
$\mathbf{R} P^{2 n+1} \subseteq(2 n+k+1)$-cf. [12, (5.2)]. When $F=\mathbf{R}, 1(\mathrm{~b})$,(c) answer affirmatively a question raised in [2] (for which, I understand, Professors Feder and Gitler also have a proof); we now show the converse is not true.

2. Example. Let $n$ be a power of 2 . Then by [8], $\mathbf{C} P^{n} \subset(4 n-1)$; 1 (b) now implies the existence of a $\mathbf{C}$-symmaxial (and so $\mathbf{R}$-symmaxial) map of type $(2 n+1,2 n-1)$. But [9], [10] $\mathbf{R} P^{2 n+1} \not \subset(4 n)$, so that the existence of $a$ symmaxial map of type $(n, k)$ does not imply $\mathbf{R} P^{n} \subset(n+k)$.

The next result is perhaps more predictable. Nevertheless, it illustrates the falsity of the converse to $[12,(5.2)]$.

3. Example. Let $n+1=2^{r}$, where $r \equiv 2,3$ (4). Then by [4] $\mathbf{R} P^{2 n+1}$ $\subseteq(4 n-2 r)$; so by [11] there exists an R-axial map of type $(2 n+1,2 n-$ $2 r-1)$. However, by [13], $\mathbf{C} P^{n} \&(4 n-2 r-1)$, whence, from 1(c), the existence of an $\mathbf{R}$-axial map of type $(2 n+1, k)$ does not imply the existence of a C-axial map of type $(2 n+1, k)$.

Since 1 shows that the situation for $\mathbf{R} P^{n}$ largely carries over to $\mathbf{C} P^{n}$, one might naively hope that a comparable result holds for $\mathbf{H} P^{n}$. However, $[3, \S 4]$ casts doubt upon, and 5 below puts paid to, such hopes.

4. Lemma. If there exists an $\mathbf{H}$-axial (resp. H-symmaxial) map $f$ of type $(4 n+3, k)$, then there exists a C-axial (resp. C-symmaxial) inap $g$ of type $(4 n+3, k)$.

Proof. Write $\mathbf{R}^{4 n+4}=\mathbf{C}^{2 n+2} \oplus \mathbf{C}^{2 n+2}$ which we identify with $\mathbf{H}^{n+1}$ as $\mathbf{C}^{2 n+2} \oplus \mathbf{C}^{2 n+2} j$. For $x_{i}, y_{i} \in \mathbf{C}^{2 n+2}, i=1,2, f$ induces $g$ by setting

$$
g\left( \pm\left(x_{1}, x_{2}\right), \pm\left(y_{1}, y_{2}\right)\right)=f\left( \pm\left(x_{1}+\bar{x}_{2} j\right), \pm\left(y_{1}+\bar{y}_{2} j\right)\right),
$$

since $\left(x_{1} a+\left(\overline{x_{2} a}\right) j\right)=\left(x_{1}+\bar{x}_{2} j\right) a$ for $a \in \mathbf{C}^{*}$. If $f$ is symmaxial then clearly $g$ is too.

5. ExAmple. Let $n$ be a power of 2. From [8], $\mathbf{H} P^{n} \subset(8 n-3)$. But if there were an $\mathbf{H}$-symmaxial-or even $\mathbf{H}$-axial-map of type $(4 n+3,4 n-3)$, then by 4 above there would exist a C-axial map of type $(4 n+3,4 n-3)$. So by 1(c) $\mathbf{C} P^{2 n+1} \subseteq(8 n-1)$, which is contradicted by [1], [13]. Hence, $\mathbf{H} P^{n} \subset$ $(4 n+k)$ does not imply the existence of an H-axial map of type $(4 n+3, k)$.

As for positive results in the quaternionic case, we must content ourselves with the following observation.

6. NotE. If there exists an $\mathbf{H}$-axial map of type $(4 n+3, k)$ with $2 k \geqslant 4 n+$ 1 , then $\mathbf{H} P^{n} \subseteq(4 n+3+k)$. The proof is as for $1(c)$ above, save that one uses the characterisation of the tangent bundle given in $[3, \S 4]$.

\section{REFERENCES}

1. J. Adem and S. Gitler, Secondary characteristic classes and the immersion problem, Bol. Soc. Mat. Mexicana (2) 8 (1963), 53-78. MR 29 \#5255.

2. J. Adem, S. Gitler and I. M. James, On axial maps of a certain type, Bol. Soc. Mat. Mexicana (2) 17 (1972), 59-62.

3. M. F. Atiyah, Thom complexes, Proc. London Math. Soc. (3) 11 (1961), 291-310. MR 24 \#A 1727

4. S. Gitler and M. Mahowald, Some immersions of real projective spaces, Bol. Soc. Mat. Mexicana (2) 14 (1969), 9-21. MR41 \#2696. 
5. A. Haefliger and M. W. Hirsch, Immersions in the stable range, Ann. of Math. (2) 75 (1962), 231-241. MR 26 \#84.

6. M. W. Hirsch, Immersions of manifolds, Trans. Amer. Math. Soc. 93 (1959), 242-276. MR 22 \#9980.

7. D. Husemoller, Fibre bundles, McGraw-'Yill, New York, 1966. MR 37 \#821.

8. I. M. James, Some embeddings of projective spaces, Proc. Cambridge Philos. Soc. 55 (1959), 294-298. MR 22 \#236.

9. J. Levine, Embedding and immersion of real projective spaces, Proc. Amer. Math. Soc. 14 (1963), 801-803. MR 27 \#272.

10. M. Mahowald, On the embeddability of the real projective spaces, Proc. Amer. Math. Soc. 13 (1962), 763-764. MR 26 \#782.

11. B. J. Sanderson, A non-immersion theorem for real projective space, Topology 2 (1963), 209-211. MR 27 \#1968.

12. Immersions and embeddings of projective spaces, Proc. London Math. Soc. (3) 14 (1964), 137-153, MR 29 \#2814.

13. B. J. Sanderson and R. L. E. Schwarzenberger, Non-immersion theorems for differentiable manifolds, Proc. Cambridge Philos. Soc. 59 (1963), 319-322. MR 26 \#5589.

St. JohN's College, OXFord OX13JP, ENGLAND 